\title{
Lower bound of the number of the Rayleigh resonances for arbitrary body
}

\author{
Plamen Stefanov \\ Department of Mathematics \\ East Carolina University \\ Greenville, NC 27858, USA
}

\begin{abstract}
We study the system of linear elasticity in an exterior domain in $\mathbf{R}^{3}$ with Neumann boundary conditions. We prove an optimal lower bound for the asymptotic distribution of the resonances near the real axis due to the existence of Rayleigh waves. To this end we construct real quasimodes as the eigenvalues of an elliptic first order UDO on the boundary. In case of a convex boundary, we prove that the resonance states are asymptotically close to the quasimode states and we use this to find explicitly the leading term of the resonance states (the Rayleigh waves) on the boundary.
\end{abstract}

\section{Introduction}

Let $\Omega \subset \mathbf{R}^{3}$ be a domain with a compact complement $\mathcal{O}$ and a smooth boundary $\Gamma$. Denote by

$$
\Delta_{e} v:=\mu_{0} \Delta v+\left(\lambda_{0}+\mu_{0}\right) \nabla(\nabla \cdot v)
$$

the operator of elasticity in $\mathbf{R}^{3}$, where $v$ is a vector-valued function. Here $\lambda_{0}, \mu_{0}$ are the Lamé constants and as usual we assume that $\mu_{0}>0,3 \lambda_{0}+2 \mu_{0}>0$. We are interested in the Neumann problem for $\Delta_{e}$ in $\Omega$. The Neumann boundary conditions for $\Delta_{e}$ require that the normal components of the stress tensor $\sigma_{i j}(v)=\lambda_{0} \nabla \cdot v \delta_{i j}+\mu_{0}\left(\frac{\partial v_{i}}{\partial x_{j}}+\frac{\partial v_{j}}{\partial x_{i}}\right)$ vanish on $\Gamma$, i.e.,

$$
\left.\sum_{j=1}^{3} \sigma_{i j}(v) n_{j}\right|_{\Gamma}=0, \quad i=1,2,3
$$

where $n(x)$ is the outer normal to $\Gamma$. Let $L$ be the self-adjoint realization of $-\Delta_{e}$ in $L^{2}(\Omega)$ with the Neumann boundary conditions (1). Throughout this paper all norms will be in $L^{2}(\Omega)$ unless otherwise stated. Resonances of $L$ are defined as usual as the poles of the meromorphic continuation $R(\lambda)$ of the resolvent $\left(L-\lambda^{2}\right)^{-1}: L_{\text {comp }}^{2}(\Omega) \rightarrow L_{\text {loc }}^{2}(\Omega)$ from 
$\operatorname{Im} \lambda<0$ to the whole complex plane $\mathbf{C}$. They are also the poles of the meromorphic continuation $R_{\chi}(\lambda)$ of the cut-off resolvent $\chi R(\lambda) \chi$ where $\chi$ is any smooth cut-off function equal to 1 near $\Gamma$. The first main result of this paper is the following.

Theorem 1.1 There exists a function $0<S(t)=O\left(t^{-\infty}\right)$, as $t \rightarrow \infty$, such that for the counting function

$$
N(r):=\#\{\lambda \text { is a resonance; } 1<\operatorname{Re} \lambda<r, 0<\operatorname{Im} \lambda<S(|\lambda|)\},
$$

$r>1$, we have

$$
N(r) \geq \frac{\operatorname{Area}(\Gamma)}{4 \pi c_{R}^{2}} r^{2}+O(r)
$$

Existence of resonances close to the real axis for the elasticity system is closely related to the existence of Rayleigh waves [R]. It is well-known [T1] that in case of Neumann boundary conditions, one has singularities propagating not only with the two sound speeds $c_{1}=\sqrt{\mu_{0}}$ and $c_{2}=\sqrt{\lambda_{0}+2 \mu_{0}}$, but a third type of singularities (Rayleigh waves) that propagate along the boundary with a slower speed $c_{R}$. This makes any obstacle a trapping one and one could expect "almost real" resonances by the Modified Lax-Phillips Conjecture. Lack of an exponential local energy decay was proved by M. Kawashita in [K1]. Existence of resonances converging rapidly to the real axis was proved by the author and G. Vodev in [SV1] for strictly convex obstacles (see also [K2] for more general systems) and next in [SV2] for obstacles with any geometry. In case of an analytic boundary the rate of convergence is exponential $[\mathrm{V}]$. An asymptotic of the counting function of the Rayleigh resonances for a class of non-trapping obstacles was established by J. Sjöstrand and G. Vodev in [SjV]. The asymptotic of the scattering phase was studied in [CV1], [CV2].

We notice that for some (trapping) obstacles there might be more resonances near the real axis. For example, if there is an elliptic periodic ray, reflecting off the boundary according to the laws of geometric optics, one might expect a lower bound $c_{0} r^{3}, c_{0}>0$ similarly to the wave equation case [S]. The bound that we get in Theorem 1.1, however, is optimal for the Rayleigh resonances because it coincides with the leading term in the asymptotic of $N(r)$ in case of a convex body $[\mathrm{SjV}]$.

Theorem 1.1 is a consequence of the existence of real Rayleigh quasimodes associated to $L$ and the result in $[\mathrm{S}]$.

\section{Theorem 1.2}

(a) There exists a sequence of positive numbers (quasimodes) $\lambda_{j} \rightarrow \infty$ and a sequence of smooth functions $u_{j} \in D(L)$ (quasimode states) supported in a fixed neighborhood of $\Gamma$ in $\Omega$ such that $\left(L-\lambda_{j}^{2}\right) u_{j}=O\left(\lambda_{j}^{-\infty}\right),\left\|u_{j}\right\|_{L^{2}(\Omega)}=1$ and $\left.u_{j}\right|_{\Gamma}$ are orthogonal up to an error $O\left(\lambda^{-\infty}\right)$ w.r.t. a suitably chosen norm in $L^{2}(\Gamma)$ equivalent to the original one.

(b) $\lambda_{j}, j=1,2, \ldots$ are the eigenvalues (repeated according to their multiplicities) of a classical first order $\Psi D O \mathcal{P}$ on $\Gamma$ coinciding with $c_{R}\left(-\Delta_{\Gamma}\right)^{1 / 2}$ modulo zero order $\Psi$ DOs. 
(c) We have $\left\|u_{j}\right\|_{L^{2}\left(\Gamma_{t}\right)} \leq C e^{-\gamma \lambda_{j} t}\left\|u_{j}\right\|_{L^{2}(\Gamma)}, 0 \leq t \ll 1$, with $\Gamma_{t}:=\{\operatorname{dist}\{x, \Gamma\}=t\}$ and any $\gamma<\left(c_{R}^{-2}-c_{1}^{-2}\right)^{1 / 2}, C=C(\gamma)$. Modulo a normalizing factor (independent of $j$ ), $u_{j}$ have the following asymptotic on $\Gamma$ :

$$
\left.\lambda_{j}^{-1 / 2} u_{j}\right|_{\Gamma}=\left(1-\frac{c_{R}^{2}}{2 \mu_{0}}\right) \lambda_{j}^{-1} \nabla_{\Gamma} \phi_{j}+\left(c_{R}^{-2}-c_{2}^{-2}\right)^{1 / 2} n(x) \phi_{j}+O\left(\lambda_{j}^{-1}\right),
$$

where $\phi_{j}$ are the normalized eigenfunctions of $\mathcal{P}$.

In order to construct the Rayleigh quasimodes whose existence is stated in Theorem 1.2, we solve the equation $\mathcal{N}(\lambda) \varphi=O\left(\lambda^{-\infty}\right) \varphi$, where $\mathcal{N}(\lambda)$ is the Neumann operator (the Dirichlet-to-Neumann map) on the boundary (see (5)). To this end, we represent $\mathcal{N}(\lambda)$ as a $\Psi D O$ with large parameter $\lambda(\lambda-\Psi D O)$ in the elliptic region in $T^{*} \Gamma$. Next, we diagonalize explicitly $\mathcal{N}(\lambda)$ in a neighborhood of the characteristic variety $\Sigma$ responsible for the Rayleigh waves. This does not only allows us to construct quasimodes, but it gives us an explicit expression of the principal term of the quasimode functions $u_{j}$ in Theorem 1.2(c). Notice that Theorem 1.2 holds for the interior problem, too.

Rayleigh quasimodes were first constructed by F. Cardoso and G. Popov in [CP]. They considered an elliptic periodic geodesic on the boundary and constructed quasimodes supported essentially near that geodesic. Their construction gives us more precise description of the support of the quasimode state but is limited to the case above.

In order to derive Theorem 1.1 from Theorem 1.2, we exploit the link between quasimodes and resonances established in [S] that generalized earlier results in [SV2], [TZ]. According to $[\mathrm{S}]$, the main term in the asymptotic of the counting function of the resonances near the real axis is bounded below by that of the quasimodes. On the other hand, the asymptotic of the quasimodes is given by the classical Weyl asymptotic of the eigenvalues of $\mathcal{P}$.

In general, one cannot expect the resonance states (and more generally, the functions in the range of the residuum of the resolvent at the resonances) to be small perturbations of the quasimode functions $u_{j}$ and therefore to have the same asymptotic on $\Gamma$. It may happen (see also [S] for a discussion of this) that we have other resonances near the real axis caused by elliptic broken rays, for example. Then the resonance states might be perturbations of linear combinations of Rayleigh and non-Rayleigh quasimode states. In the case where $\mathcal{O}$ is convex however, we can prove that the resonance states are close to linear combinations of quasimode states. This allows us to find an expression for the leading term of the Rayleigh waves at the boundary. Recall that in this case all resonances in $\Lambda:=\{\lambda \in \mathbf{C} ; 0<\operatorname{Im} \lambda<$ $\left.C_{1} \ln |\operatorname{Re} \lambda|,|\operatorname{Re} \lambda|>C_{2}\right\}$, where $C_{1}>0$ is arbitrary, $C_{2}=C_{2}\left(C_{1}\right)>0$, converge rapidly to the real axis [SV1] and as shown in [SjV], $N(r)=\left(4 \pi c_{R}^{2}\right)^{-1} \mathrm{Area}(\Gamma) r^{2}+O(r)$, i.e., the lower bound in Theorem 1.1 turns into an asymptotic.

Theorem 1.3 Let $\mathcal{O}$ be strictly convex. Then

(a) For a suitably chosen $C_{0}>0$ there exists a bijection $\rho_{j}=l\left(\lambda_{j}\right)$ between the quasimodes $\lambda_{j}$ (eigenvalues of $\mathcal{P}$ ) with $\lambda_{j}>C_{0}$ and the resonances $\rho_{j}$ in $\Lambda$ with $\operatorname{Re} \lambda_{j}>C_{0}$ such that $\rho_{j}=\lambda_{j}+O\left(\lambda_{j}^{-\infty}\right)$. 
(b) For any resonance $\rho_{j}$ as above, and any non-zero $v_{j}$ in the range of the singular part of $R(\lambda)$ at $\lambda=\rho_{j}$, we have $\left\|v_{j}\right\|_{L^{2}\left(\Gamma_{t}\right)}=O\left(\left(\left|\rho_{j}\right| t\right)^{-\infty}\right)\left\|v_{j}\right\|_{L^{2}(\Gamma)}, 0<t \ll 1$, and

$$
\left.\rho_{j}^{-1 / 2} v_{j}\right|_{\Gamma}=\left(1-\frac{c_{R}^{2}}{2 \mu_{0}}\right) \rho_{j}^{-1} \nabla_{\Gamma} \Phi_{j}+\left(c_{R}^{-2}-c_{2}^{-2}\right)^{1 / 2} n(x) \Phi_{j}+O\left(\left|\rho_{j}\right|^{-1}\right),
$$

modulo a normalizing factor, where $\Phi_{j}$ is a normalized linear combination of eigenfunctions of $\mathcal{P}$ with eigenvalues in a neighborhood of $\rho_{j}$ of size $O\left(\left|\rho_{j}\right|^{-\infty}\right)$.

This paper is organized as follows. In Section 2 we diagonalize the parametrix of the Neumann operator in the elliptic region thus reducing the construction of quasimodes to solving $(P(\lambda)-\lambda) u=O\left(\lambda^{-\infty}\right) u, \lambda \in \mathbf{R}$ on $\Gamma$ with a scalar $\lambda-\Psi$ DO $P(\lambda)$. In section 3 we show that one can replace $P(\lambda)$ by a self-adjoint classical $\Psi$ DO $\mathcal{P}$ independent of $\lambda$ and this gives us opportunity to define the quasimodes as the eigenvalues of $\mathcal{P}$ in Section 4 , where we also complete the proofs of the main theorems.

Acknowledgments. Thanks are due to the referee, whose remarks contributed significantly to improving the quality of this paper.

\section{Diagonalization of the Neumann operator}

Denote by $\mathcal{N}=\mathcal{N}(\lambda)$ the operator that maps the Dirichlet data on $\Gamma$ of an outgoing solution to its Neumann boundary data (see (5) below). Since the resonances in $\Lambda$ are among the poles of $\mathcal{N}^{-1}(\lambda)$ [SV1], constructing quasimodes of $L$ can be reduced to solving $\mathcal{N}(\lambda) f=O\left(|\lambda|^{-\infty}\right) f$. That is why we will study first $\mathcal{N}(\lambda)$ in more detail.

We are going to recall the construction of the parametrix $N_{e}$ of $\mathcal{N}$ in the elliptic region. For more details, see [G] for a similar construction for the wave equation, and [CP], [SV1], [SV2] for the elasticity system.

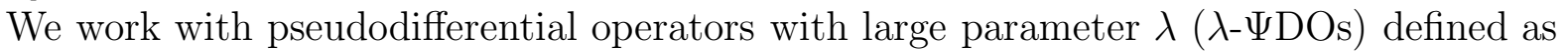
follows. In local coordinates, we have

$$
(\mathrm{Op}(a) u)(x, \lambda)=\left(\frac{\lambda}{2 \pi}\right)^{n} \iint e^{i \lambda(x-y) \cdot \xi} a(x, y, \xi, \lambda) u(y, \lambda) d y d \xi
$$

where the amplitude $a(x, y, \xi, \lambda)$ belongs to the class $S^{m, k}$, i.e., it satisfies the estimate

$$
\left|\partial_{x}^{\alpha} \partial_{y}^{\beta} \partial_{\xi}^{\gamma} a\right| \leq C_{\alpha, \beta, \gamma, K}|\lambda|^{k}(1+|\xi|)^{m-|\gamma|}
$$

for $0 \leq \operatorname{Im} \lambda \leq c, \operatorname{Re} \lambda>0,(x, y) \in K \subset \subset \mathbf{R}^{n} \times \mathbf{R}^{n}, \xi \in \mathbf{R}^{n}$. Operator (3) is well defined for $\lambda \in \Lambda$ as well if the amplitude $a$ is compactly supported with respect to $\xi$ [SV1]. Without this requirement, (3) is also well defined either by considering $\operatorname{Re} \lambda$ as the large parameter (see [SV1], [SV2]) or by using almost analytic extension (see $[\mathrm{SjV}]$ ). In this paper we are going to work with compactly supported amplitudes only and we do not need to consider $\lambda \in \Lambda$; 
instead we will assume that $0 \leq \operatorname{Im} \lambda \leq 1$ in order to cover neighborhoods of the real axis of the form $0 \leq \operatorname{Im} \lambda \leq|\lambda|^{-k}, k>0$. Because of the compact support with respect to $\xi$, the order $m$ with respect to $\xi$ does not matter and can be assumed to be $m=0$. If $a \in S^{m, k}$, then we say that $\operatorname{Op}(a) \in \mathrm{L}^{m, k}$. In fact, all amplitudes below will have asymptotic expansions $a \sim \sum_{j=-k}^{\infty} \lambda^{-j} a_{j}(x, \xi)$. We refer to $[\mathrm{G}],[\mathrm{SjV}]$ for definition of wave front set $\mathrm{WF}_{\lambda}(f)$ of

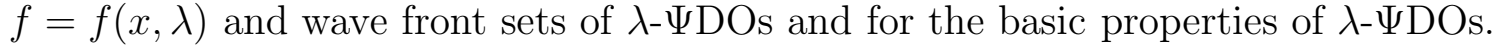

Define the Neumann operator as follows

$$
\mathcal{N}(\lambda):\left.H^{s}(\Gamma) \ni f \mapsto \sum_{j=1}^{3} \boldsymbol{\sigma}_{j}(v) n_{j}\right|_{\Gamma} \in H^{s-1}(\Gamma), \quad s \geq \frac{3}{2},
$$

where $\boldsymbol{\sigma}_{j}={ }^{t}\left(\sigma_{1 j}, \sigma_{2 j}, \sigma_{3 j}\right), \sigma_{i j}$ is the stress tensor (see (1)) and $v$ solves the following problem

$$
\left\{\begin{aligned}
\left(\Delta_{e}+\lambda^{2}\right) v & =0 & & \text { in } \Omega, \\
v & =f & & \text { on } \Gamma, \\
v & & \text { outgoing. } &
\end{aligned}\right.
$$

We will use the notation $v=\mathcal{H}(\lambda) f$.

The cotangent bundle $T^{*} \Gamma$ can be naturally decomposed into a hyperbolic, mixed, elliptic and two glancing regions with respect to the wave speeds $c_{1}=\sqrt{\mu_{0}}$ and $c_{2}=\sqrt{\lambda_{0}+2 \mu_{0}}$ of $L$ (see [SV1], for example). To construct a parametrix $N_{e}(\lambda)$ of $\mathcal{N}(\lambda)$ in the elliptic region $c_{1}\left|\xi_{x}\right|>1$, where $|\xi|_{x}$ is the norm of the covector $(x, \xi)$, we proceed as follows. In local coordinates, pick up a function $\chi \in C_{0}^{\infty}\left(T^{*} \Gamma\right)$ supported near a point in the elliptic region. Then we find an asymptotic solution of the Dirichlet problem with boundary data $\operatorname{Op}(\chi) f$ (see $(17))$ and define $N_{e}(\lambda) f$ to be the normal component of the stress tensor of that solution on the boundary. Using a partition of unity, we construct $N_{e}$ as a $\lambda-\Psi D O$ with symbol supported in the elliptic region. It is known that $N_{e}$ is elliptic outside the characteristic variety

$$
\Sigma:=\left\{(x, \xi) \in T^{*} \Gamma ; c_{R}|\xi|_{x}=1\right\}
$$

and has a simple zero on $\Sigma$. Here $c_{R}$ is the Rayleigh speed (see (9)) and $0<c_{R}<c_{1}<c_{2}$. In fact, the principal symbol of $N_{e}$ is a Hermitian matrix with three distinct and therefore smooth eigenvalues near $\Sigma$; two of them are elliptic, one of them vanishes on $\Sigma$. Using Green's formula, it is easy to see that $N_{e}$ is self-adjoint for real $\lambda$ modulo $O\left(\lambda^{-\infty}\right)$.

In [SV2] we proved that the parametrix $N_{e}(\lambda)$ and the exact solution operator $\mathcal{N}(\lambda)$ are connected via the following identity

$$
\mathcal{N}\left(f+R_{1} f\right)=N_{e} f+R_{2} f
$$

for any $f=f(x, \lambda)$ with $\|f(\cdot, \lambda)\|$ temperate in $\lambda, \mathrm{WF}_{\lambda}(f)$ supported near $\Sigma$, where $R_{1} f=$ $R_{1}(\lambda) f=O\left(|\lambda|^{-\infty}\right)$ and $R_{2}=R_{2}(\lambda) \in \mathrm{L}^{-\infty,-\infty}$. Here and below $g=O\left(|\lambda|^{-\infty}\right)$ refers to any $H^{k}$ norm of $g$ unless otherwise stated. Since we do not assume that $\mathcal{O}$ is convex, $\mathcal{N}$ may not have such a parametrix in the other regions.

In what follows we study the principal symbol $\sigma_{p}\left(N_{e}\right)$ of $N_{e}$ in more detail. We diagonalize it explicitly by finding simple formulas for the eigenvalues and the eigenvectors. We also show 
that $\sigma_{p}\left(N_{e}\right)$ admits a global (near $\Sigma$ ) diagonalization, a fact that is far from obvious. Indeed, it is easy to see that one can diagonalize $\sigma_{p}\left(N_{e}\right)$ in a neighborhood of each point in $\Sigma$ with unitary matrices depending smoothly on $(x, \xi) \in T^{*} \Gamma$. However, this does not readily imply that one can do the same thing globally near $\Sigma$ due to possible topological obstructions. Below we prove in particular that such obstructions do not exists.

Choose local coordinates $x=\left(x^{\prime}, x_{3}\right) \in \mathbf{R}^{2} \times \mathbf{R}$ as follows: fix a point $p^{0} \in \Gamma$, and choose Euclidean coordinates $\left(p_{1}, p_{2}, p_{3}\right)=\left(p^{\prime}, p_{3}\right)$, such that $p^{0}=0$ and $\Gamma$ is given by the equation $p_{3}=F\left(p^{\prime}\right)$, where $F(0)=0$ and $\nabla F(0)=0$. Then set $x_{1}=p_{1}-F\left(p^{\prime}\right), x^{\prime}=p^{\prime}$ near $p^{0}$. We can alway assume that $\Omega$ is given locally by $x_{3}>0$ Then $x^{\prime}=\left(x_{1}, x_{2}\right)$ are local coordinates on $\Gamma$ and in what follows we are going to drop the prime in order to simplify the notation. Assume that we have local coordinates as above, let $\left(x_{0}, \xi_{0}\right) \in T^{*} \Gamma$ be in the elliptic region and let us work in a neighborhood of that point.

To calculate the principal symbol $N_{e}$ we will proceed as in $[\mathrm{CP}$, sec. 2] (see also [N, sec. 7]). Set

$$
\alpha:=\left(|\xi|_{x}^{2}-\frac{1}{\mu_{0}}\right)^{1 / 2}, \quad \beta:=\left(|\xi|_{x}^{2}-\frac{1}{\lambda_{0}+2 \mu_{0}}\right)^{1 / 2}
$$

Using the explicit form of the eigenvectors of the symbol of $L$, we are looking for a solution of the form $u=\nabla v_{1}+\left(0,-\partial_{3}, \partial_{2}\right) v_{2}+\left(-\partial_{3}, 0, \partial_{1}\right) v_{3}$, where $v_{j}, j=1,2,3$ are scalar functions. Then as in $[\mathrm{CP}]$ we get that $\sigma_{p}\left(N_{e}\right)=A R^{-1}$, where

$$
A=-\lambda^{2}\left(\begin{array}{ccc}
2 i \mu_{0} \beta \xi_{1} & \mu_{0} \xi_{1} \xi_{2} & \mu_{0}\left(\xi_{1}^{2}+\alpha^{2}\right) \\
2 i \mu_{0} \beta \xi_{2} & \mu_{0}\left(\xi_{2}^{2}+\alpha^{2}\right) & \mu_{0} \xi_{1} \xi_{2} \\
1-2 \mu_{0}|\xi|^{2} & 2 i \mu_{0} \alpha \xi_{2} & 2 i \mu_{0} \alpha \xi_{1}
\end{array}\right), \quad R=i \lambda\left(\begin{array}{ccc}
\xi_{1} & 0 & -i \alpha \\
\xi_{2} & -i \alpha & 0 \\
i \beta & \xi_{2} & \xi_{1}
\end{array}\right)
$$

We thus get

$$
\sigma_{p}\left(N_{e}\right)(0, \xi)=-\lambda\left(|\xi|^{2}-\alpha \beta\right)^{-1} N_{1}
$$

where

$$
N_{1}=\left(\begin{array}{ccc}
\mu_{0}(\alpha-\beta) \xi_{2}^{2}+\beta & -\mu_{0} \xi_{1} \xi_{2}(\alpha-\beta) & -i\left(2 \mu_{0}|\xi|^{2}-2 \mu_{0} \alpha \beta-1\right) \xi_{1} \\
-\mu_{0} \xi_{1} \xi_{2}(\alpha-\beta) & \mu_{0}(\alpha-\beta) \xi_{1}^{2}+\beta & -i\left(2 \mu_{0}|\xi|^{2}-2 \mu_{0} \alpha \beta-1\right) \xi_{2} \\
i\left(2 \mu_{0}|\xi|^{2}-2 \mu_{0} \alpha \beta-1\right) \xi_{1} & i\left(2 \mu_{0}|\xi|^{2}-2 \mu_{0} \alpha \beta-1\right) \xi_{2} & \alpha
\end{array}\right) .
$$

A direct inspection of $N_{1}$ shows that $\left(-\xi_{2}, \xi_{1}, 0\right)$ is an eigenvector. Based on this observation, let us choose a unitary matrix $V$ with one column parallel to that vector:

$$
V=\left(\begin{array}{ccc}
\xi_{1} /|\xi| & 0 & -\xi_{2} /|\xi| \\
\xi_{2} /|\xi| & 0 & \xi_{1} /|\xi| \\
0 & 1 & 0
\end{array}\right)
$$

Then

$$
V^{*} N_{1} V=\left(\begin{array}{ccc}
\beta & -i\left(2 \mu_{0}|\xi|^{2}-2 \mu_{0} \alpha \beta-1\right)|\xi| & 0 \\
i\left(2 \mu_{0}|\xi|^{2}-2 \mu_{0} \alpha \beta-1\right)|\xi| & \alpha & 0 \\
0 & 0 & \mu_{0} \alpha\left(|\xi|^{2}-\alpha \beta\right) .
\end{array}\right) .
$$


Therefore, one of the eigenvalues of $\sigma_{p}\left(N_{e}\right)(0, \xi)$ is $a_{3}:=-\lambda \mu_{0} \alpha$, while the other two are the eigenvalues $m_{1}, m_{2}$ of

$$
M:=\left(\begin{array}{cc}
\beta & -i|\xi|\left(2 \mu_{0}\left(|\xi|^{2}-\alpha \beta\right)-1\right) \\
i|\xi|\left(2 \mu_{0}\left(|\xi|^{2}-\alpha \beta\right)-1\right) & \alpha
\end{array}\right)
$$

multiplied by $-\lambda\left(|\xi|^{2}-\alpha \beta\right)^{-1}$. This leads to a quadratic equation for the other two eigenvalues $a_{1}$ and $a_{2}$. Its discriminant $D:=(\alpha-\beta)^{2}+4|\xi|^{2}\left(2 \mu_{0}\left(|\xi|^{2}-\alpha \beta\right)-1\right)^{2}$ is clearly positive in the elliptic region. Therefore, we have one eigenvalue of $M$ of the kind $m_{2}:=(\alpha+\beta+\sqrt{D}) / 2$ which is always positive, while the other one $m_{1}:=(\alpha+\beta-\sqrt{D}) / 2$ may vanish. Next,

$$
\operatorname{det} M=\left(|\xi|^{2}-\alpha \beta\right)\left(4 \mu_{0}^{2}|\xi|^{2} \alpha \beta-\left(1-2 \mu_{0}|\xi|^{2}\right)^{2}\right) \text {. }
$$

The first factor is positive in the elliptic region, while the second one vanishes for $|\xi|=s$, where $s$ solves the equation

$$
4 \mu_{0}^{2} s^{2}\left(s^{2}-\frac{1}{\mu_{0}}\right)^{1 / 2}\left(s^{2}-\frac{1}{\lambda_{0}+2 \mu_{0}}\right)^{1 / 2}-\left(1-2 \mu_{0} s^{2}\right)^{2}=0 .
$$

It is well-known that there is unique simple root of this equation in the interval $s>1 / \sqrt{\mu_{0}}$ (see [K1], [T1]). Denote that root by $1 / c_{R}$. Then $c_{R}$ is the Rayleigh speed and we have that $\operatorname{det} M$ and therefore the first eigenvalue $a_{1}$ of $\sigma_{p}\left(N_{e}\right)(0, \xi)$ has a simple zero at $c_{R}|\xi|=1$. Writing this into an invariant form, we get that $\sigma_{p}\left(N_{e}\right)$ has three eigenvalues in the elliptic region, one of them vanishes on the characteristic variety $\Sigma$ and therefore has the form $a_{1}=\lambda\left(c_{R}|\xi|_{x}-1\right) a_{1}^{\prime}, a_{1}^{\prime} \neq 0$, while the other two are non-vanishing in the elliptic region. Studying the limit of $\operatorname{det} M$ as $|\xi| \rightarrow \infty$ one can show that $a_{1}^{\prime}<0$. As we mentioned above, this behavior of the eigenvalues of $N_{e}$ is a known fact. Using equation (9), we get that $\mu_{0}\left(|\xi|_{x}^{2}-\alpha \beta\right)=1-1 /\left(4 \mu_{0}|\xi|_{x}^{2}\right)$ on $\Sigma$, while the quadratic equation for $m_{1}$ and $m_{2}$ implies $m_{2}=(\alpha+\beta+\sqrt{D}) / 2=\alpha+\beta$ on $\Sigma$. Therefore, for the eigenvalues $a_{j}, j=1,2,3$ of $\sigma_{p}(N)$ we have $a_{1}=0, a_{2}=-\lambda \mu_{0}(\alpha+\beta)\left(1-1 /\left(4 \mu_{0}|\xi|_{x}^{2}\right)\right)^{-1}, a_{3}=-\lambda \mu_{0} \alpha$ on $\Sigma$. In particular, $a_{2}<a_{3}<a_{1}$ in a neighborhood of $\Sigma$, which proves that the three eigenvalues are distinct near $\Sigma$.

We are going now to calculate the eigenvectors $v_{j}, j=1,2,3$ of $\sigma_{p}\left(N_{e}\right)$. Let us first work in the local coordinates defined above. To find an eigenvector $v_{1}$ associated to the eigenvalue $a_{1}$ vanishing on $\Sigma$, we proceed as follows. By (8) we see that

$$
w_{1}:=\left(i|\xi|\left(2 \mu_{0}\left(|\xi|^{2}-\alpha \beta\right)-1\right), \beta-m_{1}\right)=\left(i|\xi|\left(2 \mu_{0}\left(|\xi|^{2}-\alpha \beta\right)-1\right), \frac{1}{2}(\beta-\alpha+\sqrt{D})\right)
$$

is an eigenvector of $M$ corresponding to $m_{1}$. One can therefore set $\tilde{w}_{1}:=\left(w_{1}, 0\right) \in \mathbf{R}^{3}$ to get an eigenvector of $V^{*} N_{1} V$. Hence $v_{1}:=V \tilde{w}_{1}$ is the eigenvector we are looking for and a direct calculation yields

$$
v_{1}(0, \xi)=\left(i \xi_{1}\left(2 \mu_{0}|\xi|^{2}-2 \mu_{0} \alpha \beta-1\right), i \xi_{2}\left(2 \mu_{0}|\xi|^{2}-2 \mu_{0} \alpha \beta-1\right), \frac{1}{2}(\beta-\alpha+\sqrt{D})\right) .
$$


Similarly,

$$
v_{2}(0, \xi)=\left(i \xi_{1}\left(2 \mu_{0}|\xi|^{2}-2 \mu_{0} \alpha \beta-1\right), i \xi_{2}\left(2 \mu_{0}|\xi|^{2}-2 \mu_{0} \alpha \beta-1\right), \frac{1}{2}(\beta-\alpha-\sqrt{D})\right) .
$$

The third eigenvector, as pointed out at the beginning, can be chosen to be

$$
v_{3}(0, \xi)=\left(-\xi_{2}, \xi_{1}, 0\right)
$$

It is easy to check that $v_{1,2} \neq 0$ near $\Sigma$ because $2 \mu_{0}|\xi|^{2}-2 \mu_{0} \alpha \beta-1=1-1 /\left(2 \mu_{0}|\xi|^{2}\right)=$ $\sqrt{\alpha \beta} /|\xi|>0$ on $\Sigma$. We claim that those three eigenvectors are defined invariantly. Notice first that $T^{*} \Gamma$ can be identified with $\left\{(x, \xi) \in \Gamma \times \mathbf{R}^{3} ; x \in \Gamma, \xi \perp n(x)\right\}$. Using this, we represent $v_{1,2}$ as $v_{1,2}=i\left(2 \mu_{0}|\xi|_{x}^{2}-2 \mu_{0} \alpha \beta-1\right) \xi+\frac{1}{2}(\beta-\alpha \pm \sqrt{D}) n(x)$. Similarly, $v_{3}=$ $n(x) \times \xi$, where $\times$ stands for the vector product in $\mathbf{R}^{3}$. Let us summarize this in the following proposition.

Proposition 2.1 The principal symbol $\sigma_{p}\left(N_{e}\right)$ of the Neumann operator in the elliptic region has three distinct eigenvalues $a_{j}, j=1,2,3$ near $\Sigma$ such that $a_{1}=\left(c_{R}|\xi|_{x}-1\right) a_{1}^{\prime}, a_{1}^{\prime}<0$ on $\Sigma$ and $a_{2}<a_{3}<0$. Furthermore, $\sigma_{p}\left(N_{e}\right)$ admits three globally defined near $\Sigma$ eigenvectors $v_{j}$, corresponding to $a_{j}, j=1,2,3$, respectively, having the form

$$
v_{1,2}=i\left(2 \mu_{0}|\xi|_{x}^{2}-2 \mu_{0} \alpha \beta-1\right) \xi+\frac{1}{2}(\beta-\alpha \pm \sqrt{D}) n(x), \quad v_{3}=n(x) \times \xi
$$

with $D=(\alpha-\beta)^{2}+4|\xi|_{x}^{2}\left(2 \mu_{0}\left(|\xi|_{x}^{2}-\alpha \beta\right)-1\right)^{2}$. On $\Sigma$ we have $\sqrt{D}=\alpha+\beta$ and

$$
v_{1}=i\left(1-\frac{c_{R}^{2}}{2 \mu_{0}}\right) \xi+\left.\beta\right|_{\Sigma} n(x), \quad v_{2}=i\left(1-\frac{c_{R}^{2}}{2 \mu_{0}}\right) \xi-\left.\alpha\right|_{\Sigma} n(x) .
$$

We are going now to diagonalize $N_{e}$ completely using essentially the construction in [T2, sec. V.5] with minor modifications making sure that the transformation operators are unitary. Let $V_{1} \subset \subset V_{2}$ be two sufficiently small neighborhoods of $\Sigma$. First we will choose a unitary operator $U_{0}$ in $V_{1}$ with "full symbol" supported in $V_{2}$ diagonalizing the principal symbol of $N_{e}$. More precisely, we require that $U^{*} U X=X$ modulo $\mathrm{L}^{-\infty,-\infty}$ for any $\lambda-\Psi \mathrm{DO}$ $X$ with symbol supported in $V_{1}$. Note that although the full symbol of a $\lambda-\Psi D O$ is not

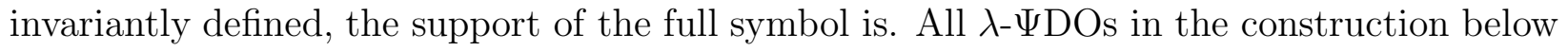
will be assumed to have full symbols supported in $V_{2}$. In order to simplify the notation we will omit operators like $X$ below and $A=B$ will mean that for any $X$ as above we have $A X=B X+R_{\infty}$ with $R_{\infty} \in \mathrm{L}^{-\infty,-\infty}$. This is equivalent to the assumption that instead of working with operators, we work with symbols and $A=B$ means that the symbols of $A$ and $B$ coincide in $V_{1}$ modulo negligible ones. We can construct $U$ as follows. First, choose $\tilde{U}$ with principal symbol

$$
\sigma_{p}(\tilde{U}):=\left(\frac{v_{1}}{\left|v_{1}\right|_{x}}, \frac{v_{2}}{\left|v_{2}\right|_{x}}, \frac{v_{3}}{\left|v_{3}\right|_{x}}\right) .
$$

Then $\sigma_{p}(\tilde{U})$ is a unitary matrix in $V_{1}$. Therefore, $\tilde{U}^{*} \tilde{U}=1+R$ with $R$ self-adjoint of order -1 . Set $U_{0}:=\tilde{U}(1+R)^{-1 / 2}$. The square root here can be defined as a $\lambda-\Psi$ DO by using the 
Taylor expansion of $z \mapsto(1+z)^{-1 / 2}$ near $z=0$ and setting $z=R(\lambda)=O\left(|\lambda|^{-1}\right)$ for $|\lambda| \gg 1$ in that expansion. Then $U_{0}$ has the required properties and the same principal symbol as $\tilde{U}$. Moreover, $U_{0}$ is invertible for large $\lambda$.

Clearly $U_{0}$ diagonalizes the principal symbol of $N_{e}$. We are going to work next with matrix-valued operators $T$ written in the following block form

$$
T:=\left(\begin{array}{ll}
T_{11} & T_{12} \\
T_{21} & T_{22}
\end{array}\right)
$$

where $T_{11}$ is a scalar $\lambda$ - $\Psi \mathrm{DO}, T_{12}$ is $1 \times 2, T_{21}$ is $2 \times 1, T_{22}$ is $2 \times 2$. With this notation, we have

$$
U_{0}^{*} N_{e} U_{0}=A+B:=\left(\begin{array}{cc}
A_{1}^{\prime}\left(c_{R}\left(1-\Delta_{\Gamma}\right)^{\frac{1}{2}}-\lambda\right) A_{1}^{\prime} & 0 \\
0 & A_{2}
\end{array}\right)+\left(\begin{array}{cc}
B_{11} & B_{12} \\
B_{21} & B_{22}
\end{array}\right) .
$$

where $A_{1}^{\prime}$ is a scalar elliptic $\lambda$ - $\Psi$ DO of order 0 with $\sigma_{p}\left(A_{1}^{\prime}\right)=\left(a_{1}^{\prime}\right)^{1 / 2}, A_{2}$ is a $2 \times 2$ diagonal

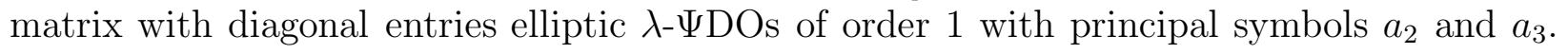
The remainder $B$ is of order 0 . Let us fix some self-adjoint (for real $\lambda$ ) $A_{1}^{\prime}, A_{2}$ with those properties. Then $B$ is self-adjoint for real $\lambda$ modulo $\mathrm{L}^{-\infty,-\infty}$.

After diagonalizing $N_{e}$ modulo operators of order 0 , next step will be to kill the offdiagonal entries of $B$ modulo operators of order -1 . We are looking for a $\lambda-\Psi D O R_{1}$ of order -1 such that $\left(1+R_{1}\right)^{-1}(A+B)\left(1+R_{1}\right)$ is in a diagonal block form modulo $\mathrm{L}^{0,-1}$. Assume that

$$
R_{1}:=\left(\begin{array}{cc}
0 & -\left(R_{1}^{*}\right)_{21} \\
\left(R_{1}\right)_{21} & 0
\end{array}\right)
$$

Then $\left(1+R_{1}\right)^{-1}(A+B)\left(1+R_{1}\right)=A+\left(A R_{1}-R_{1} A+B\right) \bmod \mathrm{L}^{0,-1}$. Therefore, the off-diagonal entries of $\left(1+R_{1}\right)^{-1}(A+B)\left(1+R_{1}\right)$ of order 0 will be killed if $\left(R_{1}\right)_{21}$ solves

$$
\left(R_{1}\right)_{21} A_{1}-A_{2}\left(R_{1}\right)_{21}=B_{21}, \quad-\left(R_{1}\right)_{21}^{*} A_{2}+A_{1}\left(R_{1}\right)_{21}^{*}=B_{12}
$$

Here $A_{1}:=A_{1}^{\prime}\left(c_{R}\left(1-\Delta_{\Gamma}\right)^{\frac{1}{2}}-\lambda\right) A_{1}^{\prime}$. The second equation is just the adjoint to the first one. Since $\sigma_{p}\left(A_{1}\right)$ and the diagonal entries of $\sigma_{p}\left(A_{2}\right)$ are distinct in $V_{2}$, the solvability of (11) on the principal symbol level follows from [T2, Ch. IX, Lemma 1.1]. Fix $R_{1}$ with such a principal symbol. Note that $1+R_{1}$ may not be unitary, because $\left(1+R_{1}\right)^{*}\left(1+R_{1}\right)=1+R_{1}^{*} R_{1}$. Set

$$
U_{1}:=\left(1+R_{1}\right)\left(1+R_{1}^{*} R_{1}\right)^{-1 / 2}
$$

Then $U_{1}$ is unitary and $U_{1}=1+R_{1} \bmod \mathrm{L}^{0,-2}$. Therefore, after replacing $1+R_{1}$ by $U_{1}$, operator $U_{1}^{*}(A+B) U_{1}$ still has the diagonal form we need $\bmod \mathrm{L}^{0,-1}$. So after this step we obtain

$$
U_{1}^{*} U_{0}^{*} N_{e} U_{0} U_{1}=\left(\begin{array}{cc}
A_{1}^{\prime}(\tilde{P}-\lambda) A_{1}^{\prime} & 0 \\
0 & \tilde{Q}
\end{array}\right)+\left(\begin{array}{ll}
C_{11} & C_{12} \\
C_{21} & C_{22}
\end{array}\right)
$$

with $\tilde{P}=c_{R}\left(1-\Delta_{\Gamma}\right)^{\frac{1}{2}} \bmod \mathrm{L}^{0,0}, \tilde{Q}=A_{2} \bmod \mathrm{L}^{0,0}$ and $C \in \mathrm{L}^{0,-1}$, all of them self-adjoint for real $\lambda$ modulo negligible operators. Proceeding in the same way, we find unitary operators $U_{2}, U_{3}, \ldots$ such that $\left(U_{0} \ldots U_{k-1} U_{k}\right)^{*} N_{e}\left(U_{0} \ldots U_{k-1} U_{k}\right)$ is in diagonal form mod $\mathrm{L}^{0,-k}$. Using 
a standard argument, choose $U \sim U_{0} U_{1} U_{2} \ldots$ Then $U$ will be unitary mod $\mathrm{L}^{0,-\infty}$ and $U^{*} N_{e} U=\operatorname{diag}\left(A_{1}^{\prime}(P-\lambda) A_{1}^{\prime}, Q\right)$. We can arrange that $U$ is unitary (exactly) by using arguments similar to those above. From our construction it follows that $P=c_{R}\left(1-\Delta_{\Gamma}\right)^{\frac{1}{2}}$ $\bmod \mathrm{L}^{0,0}$. Since $N_{e}$ is self-adjoint modulo $\mathrm{L}^{0,-\infty}$ for real $\lambda$, we have the same for $A_{1}^{\prime}(P-\lambda) A_{1}^{\prime}$ and therefore for $P$. Thus we have proved the following.

Proposition 2.2 There exits $U \in \mathrm{L}^{0,0}$ with $U^{*} U=U U^{*}=1$ such that for any $X$ as above we have in block form

$$
U^{*} N_{e} U X=\left(\begin{array}{cc}
A_{1}^{\prime}(P-\lambda) A_{1}^{\prime} & 0 \\
0 & Q
\end{array}\right) X \quad \bmod \mathrm{L}^{-\infty,-\infty} .
$$

Here $A_{1}^{\prime} \in \mathrm{L}^{0,0}, Q \in \mathrm{L}^{1,1}$ are elliptic self-adjoint operators for real $\lambda, P \in \mathrm{L}^{1,1}$ is also selfadjoint for real $\lambda$, and $P=c_{R}\left(1-\Delta_{\Gamma}\right)^{\frac{1}{2}} \bmod \mathrm{L}^{0,0}$. Moreover, the principal symbol of $U$ near $\Sigma$ is given by (10).

\section{Reduction to a classical $\Psi$ DO.}

The construction of quasimodes is related to solving the equation $(P-\lambda) u=O\left(\lambda^{-\infty}\right) u$ for certain real $\lambda$ 's. Here $P \in \mathrm{L}^{1,1}$ depends on $\lambda$ and only its principal term does not. Here we will show that one can reduce that problem to solving a similar equation with $P$ a classical $\Psi D O$ independent of $\lambda$ with the same principal term. We refer to [PV] for a similar construction for the transmission problem. To stress on the dependence on $\lambda$ we will use the notation $P(\lambda)$ instead of $P$ and similarly for other $\lambda$ - $\Psi$ DOs.

We can think that the symbol of $P(\lambda)$ is extended from a neighborhood of $\Sigma$ to the whole $T^{*} \Gamma$ minus a small neighborhood $V$ of the zero section such that in any local coordinates for the symbol $P(x, \xi, \lambda)$ of $P(\lambda)$ we have

$$
P(x, \xi, \lambda)-\lambda \sim \lambda\left(c_{R}|\xi|_{x}-1\right)+\sum_{k=0}^{\infty} \lambda^{-k} p_{-k}(x, \xi)
$$

with $p_{-k} \in S^{-k}$. It is not hard to arrange that the so extended $P(\lambda)$ is still self-adjoint modulo negligible operators. The first step will be to reduce the problem to a similar one with polyhomogeneous symbol outside $V$, i.e., with $p_{-k}$ homogeneous of order $-k$ outside $V$. Denote by $P_{1}(\lambda)$ such an operator with principal symbol $\left(c_{R}|\xi|_{x}-1\right)$ outside $V$. Without loss of generality we can assume that $P_{1}(\lambda)$ is self-adjoint for real $\lambda$. Then

$$
P(x, \xi, \lambda)-\lambda \sim P_{1}(x, \xi, \lambda)-\lambda+\sum_{k=0}^{\infty} \lambda^{-k} \tilde{p}_{-k}(x, \xi)
$$

Since $P(\lambda)-P_{1}(\lambda)$ is self-adjoint for real $\lambda, \tilde{p}_{0}(x, \xi)$ is real-valued. We have

$$
\tilde{p}_{0}(x, \xi)=\tilde{p}_{0}\left(x, \frac{\xi}{c_{R}|\xi|_{x}}\right)+\lambda\left(c_{R}|\xi|_{x}-1\right) \frac{r_{1}(x, \xi)}{\lambda}
$$


where $r_{1}$ is smooth for $\xi \neq 0$ and real-valued. In order to avoid the singularity at $\xi=0$, let us redefine the first term in the r.h.s. above and $r_{1}$ as smooth functions near the zero section by keeping the equality above. Denote by $P_{0}(\lambda)$ an $\lambda$ - $\Psi$ DO with polyhomogeneous symbol outside $V$ with principal symbol $\tilde{p}_{0}\left(x, \xi /\left(c_{R}|\xi|_{x}\right)\right)$ for $(x, \xi) \notin V$. Let $R_{1}(\lambda)$ have principal symbol $-r_{1} /(2 \lambda)$. We can choose $P_{0}(\lambda)$ and $R_{1}(\lambda)$ to be self-adjoint for real $\lambda$. Then

$$
\left(1+R_{1}(\lambda)\right)(P(\lambda)-\lambda)\left(1+R_{1}(\lambda)\right)=P_{1}(\lambda)+P_{0}(\lambda)-\lambda+Q_{1}(\lambda)
$$

with $Q_{1} \in \mathrm{L}^{-1,-1}$ and $P_{0}$ of order 0 such that its principal symbol has the desired homogeneity property. Moreover, $Q_{1}(\lambda)$ is self-adjoint for $\lambda \in \mathbf{R}$.

We are going to complete the proof by an inductive argument. Suppose that for some $k \geq 1$ we have

$$
\left(1+\sum_{j=1}^{k} \rho_{j}(\lambda)\right)(P(\lambda)-\lambda)\left(1+\sum_{j=1}^{k} \rho_{j}(\lambda)\right)=P_{1}(\lambda)+\ldots+P_{-k+1}(\lambda)-\lambda+Q_{k}(\lambda)
$$

with $P_{-j} \in \mathrm{L}^{-j,-j}, j=-1,0,1, \ldots, k-1$ with polyhomogeneous symbol outside $V, \rho_{j} \in$ $\mathrm{L}^{-j,-j}, j=1, \ldots, k$ and $Q_{k} \in \mathrm{L}^{-k,-k}$. Assume also that all operators above are self-adjoint for $\lambda \in \mathbf{R}$. Then for the principal symbol $\lambda^{-k} q_{k}^{0}(x, \xi)$ of $Q_{k}$ we have that

$$
\lambda^{-k} q_{l}^{0}(x, \xi)-\lambda^{-k} c_{R}^{-k}|\xi|_{x}^{-k} q_{k}^{0}\left(\frac{\xi}{c_{R}|\xi|_{x}}\right)=0 \quad \text { on } \Sigma
$$

Therefore,

$$
\lambda^{-k} q_{k}^{0}(x, \xi)=\lambda^{-k} c_{R}^{-k}|\xi|_{x}^{-k} q_{k}^{0}\left(\frac{\xi}{c_{R}|\xi|_{x}}\right)+\lambda\left(c_{R}|\xi|_{x}-1\right) \lambda^{-k-1} r_{k+1}(x, \xi)
$$

with $r_{k+1}$ smooth outside $V$ and real-valued. Let us extend smoothly in $V$ both terms in the r.h.s. above by keeping the identity such that those extensions vanish in a subset of

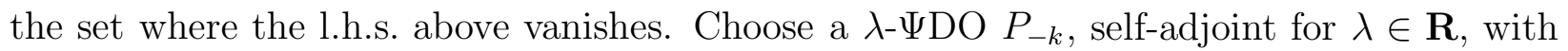
polyhomogeneous symbol outside $V$ and with principal symbol equal to the first term in the r.h.s. above. Multiplying (15) on the left and on the right by $1+R_{k+1}$, where $R_{k+1}=R_{k+1}^{*}$ for $\lambda \in \mathbf{R}$ has principal symbol $-\lambda^{-k-1} r_{k+1} / 2$, we get (15) with $k$ replaced by $k+1$. This completes our inductive argument and proves the following.

Proposition 3.1 With $X(\lambda)$ as above, there exists $R(\lambda) \in \mathrm{L}^{-1,-1}$ such that

$$
(1+R(\lambda))(P(\lambda)-\lambda)(1+R(\lambda)) X(\lambda)=(\tilde{P}(\lambda)-\lambda) X(\lambda)+Q_{\infty}(\lambda)
$$

with $\tilde{P}(\lambda)$ having a polyhomogeneous symbol outside a small neighborhood $V$ of $\xi=0$ and $Q_{\infty} \in \mathrm{L}^{-\infty,-\infty}$. Moreover, $\tilde{P}(\lambda)$ and $R(\lambda)$ are self-adjoint for $\lambda \in \mathbf{R}$. 
Next step is to reduce the problem to a similar one with $\tilde{P}(\lambda)$ independent of $\lambda$. In what follows we will say that a classical symbol $a_{\lambda}(x, y, \xi)$ belongs to the class $S^{k}$ (of classical symbols) uniformly in $\lambda$, if it is uniformly bounded in the Fréchet space $S^{k}$. If a classical $\Psi \mathrm{DO} A_{\lambda}$ has a full symbol belonging uniformly to $S^{k}$ in any local coordinates, we say that $A \in \Psi^{k}$ uniformly in $\lambda$. A similar definition applies for $k=-\infty$ (see also [PV]).

We are going to work in a neighborhood of the characteristic variety again, which in the classical pseudodifferential calculus has the form $c_{R}|\xi|_{x}=\lambda$. Below, given two classical $\Psi$ DOs depending on $\lambda$, the identity $A(\lambda)=B(\lambda)$ will mean that $A(\lambda) X(\lambda)=B(\lambda) X(\lambda)+R(\lambda)$ for some $X(\lambda) \in \Psi^{0}$ uniformly in $\lambda$ having full symbol supported near the characteristic variety, where $R(\lambda)$ is smoothing and $R(\lambda)=O\left(|\lambda|^{-\infty}\right)$. Such $X(\lambda)$ can be obtained as $\lambda$ - $\Psi$ DOs with compactly supported symbols near $\Sigma$.

For the symbol $\tilde{P}(x, \xi, \lambda)$ of $\tilde{P}(\lambda)$ we have in local coordinates

$$
\tilde{P}(x, \xi, \lambda)-\lambda \sim \lambda\left(c_{R}|\xi|_{x}-1\right)+\sum_{j=0}^{\infty} \lambda^{-j} \tilde{p}_{-j}(x, \xi) \quad \text { near } \Sigma
$$

with $\tilde{p}_{-j}$ homogeneous of order $-j$. Writing this down as a classical $\Psi$ DO we get

$$
\sigma(\tilde{P}(\lambda))-\lambda \sim c_{R}|\xi|_{x}-\lambda+\sum_{j=0}^{\infty} \tilde{p}_{-j}(x, \xi) \quad \text { near } c_{R}|\xi|_{x} / \lambda=1 .
$$

Here and below "near $|\xi|_{x} / \lambda=1$ " means that the identity above holds for $\left.\left|c_{R}\right| \xi\right|_{x}-\lambda \mid<\varepsilon \lambda$ with $\varepsilon>0$ that can vary from step to step. If $\varepsilon$ is small enough, and $\lambda>1$ then $\xi$ is outside a fixed neighborhood of the zero section, say, $|\xi|_{x}<1$ and therefore $\tilde{p}_{-j}$ are not singular there, so we can cut near $\xi=0$. Relation (16) shows that there exists $\mathcal{P} \in \Psi^{1}$ independent of $\lambda$ with principal symbol $c_{R}|\xi|_{x}$ for $|\xi|_{x}>1$, such that

$$
\tilde{P}(\lambda)-\lambda=\mathcal{P}-\lambda
$$

in the sense described above. Here we have used the fact that in $R_{0}$ is smoothing, then $R_{0} X(\lambda)=O\left(|\lambda|^{-\infty}\right)$. We have therefore proved the following.

Proposition 3.2 There exists $\varepsilon>0$ such that for any classical $\Psi D O X(\lambda) \in \Psi^{1}$ uniformly in $\lambda$ having full symbol supported in $\left.\left|c_{R}\right| \xi\right|_{x}-\lambda \mid<\varepsilon \lambda$ in any local coordinates, we have

$$
(\tilde{P}(\lambda)-\lambda) X(\lambda)=(\mathcal{P}-\lambda) X(\lambda)+R(\lambda) .
$$

Here $R(\lambda)=O\left(|\lambda|^{-\infty}\right)$ is smoothing and $\mathcal{P} \in \Psi^{1}$ with principal symbol $c_{R}|\xi|_{x}$.

Combining the last three propositions we obtain the central result in this section.

Theorem 3.1 There exits $V(\lambda) \in \Psi^{1}$ uniformly in $\lambda$, invertible for large $\lambda$ uniformly in $\lambda$, and a self-adjoint $\mathcal{P} \in \Psi^{1}$ independent of $\lambda$, such that for $N_{e}$ we have in block form

$$
V^{*}(\lambda) N_{e}(\lambda) V(\lambda) X(\lambda)=\left(\begin{array}{cc}
\mathcal{P}-\lambda & 0 \\
0 & Q(\lambda)
\end{array}\right) X(\lambda)+R(\lambda) .
$$

Here $Q(\lambda) \in \mathrm{L}^{1,1}$ is elliptic and self-adjoint for real $\lambda, R(\lambda)=O\left(|\lambda|^{-\infty}\right)$ is smoothing, and $\mathcal{P}=c_{R}\left(-\Delta_{\Gamma}\right)^{\frac{1}{2}} \bmod \Psi^{0}$. 


\section{Construction of the quasimodes; the lower bound}

We are ready to proceed with the construction of the quasimodes. Let $\phi_{j}$ and $\lambda_{j}, j=1,2, \ldots$, be the eigenfunctions and eigenvalues (counted with their multiplicities) of the scalar-valued operator $\mathcal{P}$ in $L^{2}(\Gamma)$. We assume that $\phi_{j}$ are normalized and since $\mathcal{P}=c_{R}\left(-\Delta_{\Gamma}\right)^{1 / 2} \bmod \Psi^{0}$, all $\lambda_{j}$ are positive except finitely many that we omit. Set $\varphi_{j}:=\varphi\left(\lambda_{j}\right)=V\left(\lambda_{j}\right)\left(\phi_{j}, 0,0\right)$. If we denote $\varphi=\varphi(\lambda), \lambda \in\left\{\lambda_{j}\right\}_{j=1}^{\infty}$, then $\mathrm{WF}_{\lambda}(\varphi) \subset \Sigma$. Therefore, $X(\lambda) \varphi(\lambda)=\varphi(\lambda)+O\left(\lambda^{-\infty}\right)$. Here $X(\lambda)$ is constructed as follows: near any point $\zeta \in \Sigma$ we choose $X_{\zeta}=\operatorname{Op}(\chi)$, where $\chi$ is a cut-off function equal to 1 near that point. Using a partition of unity, we construct $X(\lambda)$. Therefore, $N_{e}\left(\lambda_{j}\right) \varphi_{j}=O\left(\lambda_{j}^{-\infty}\right), j=1,2, \ldots$. The same is true for $\psi_{j}:=\varphi_{j} /\left\|\varphi_{j}\right\|$ because $V\left(\lambda_{j}\right)$ and $V^{-1}\left(\lambda_{j}\right)$ are uniformly bounded. Then $V^{-1}\left(\lambda_{j}\right) \psi_{j}, j=1,2, \ldots$, are orthogonal. Next, $\mathcal{N}\left(\lambda_{j}\right) \psi_{j}=O\left(\lambda_{j}^{-\infty}\right)$ and $\mathrm{WF}_{\lambda}\left(\left\{\psi_{j}\right\}\right) \subset \Sigma$. We define the quasimodes as asymptotic solutions to the Dirichlet problem with boundary data $\psi_{j}$, cut-off near the boundary. More precisely, the asymptotic solution (see [SV1], [SV2]) is given locally by

$$
H_{e}\left(\lambda_{j}\right) \psi_{j}=\sum_{k=1}^{2}\left(\frac{\lambda_{j}}{2 \pi}\right)^{2} \iint e^{i \lambda_{j}\left(\Phi_{k}(x, \xi)-y \cdot \xi\right)} h^{(k)}\left(x, \xi, \lambda_{j}\right) \psi_{j}(y) d y d \xi
$$

The phase functions $\Phi_{k}$ solve the eikonal equations $c_{k}^{2}\left(\nabla \Phi_{k}\right)^{2}=1,\left.\Phi_{k}\right|_{\Gamma}=x^{\prime} \cdot \xi$ to infinite order at $\Gamma$ and $\operatorname{Im} \Phi_{k}=x_{3} \sqrt{c_{R}^{-2}-c_{k}^{-2}}+O\left(x_{3}^{2}\right), k=1,2$ for $0<x_{3} \ll 1$ and $\left(x^{\prime}, \xi\right) \in \Sigma$. Here $x=\left(x^{\prime}, x_{3}\right)$ are local coordinates as in section 2 and it is now convenient to assume that $x_{3}=\operatorname{dist}(x, \Gamma)$. We get $H_{e}\left(\lambda_{j}\right) \psi_{j}=O\left(e^{-\gamma \lambda_{j} x_{3}}\right)$ with $\gamma>0$ as in Theorem 1.2. The matrixvalued amplitudes $h^{(k)}$ are solutions of the corresponding transport equations and have the form $h^{(k)}=\sum_{j=0}^{\infty} h_{j}^{(k)}(x, \xi) \lambda^{-j}$, with $h_{j}^{(k)}$ formal series in $x_{3}$ supported near $\left(x^{\prime}, \xi\right) \in \Sigma$. Let $\chi$ be a cut-off function equal to one near $\Gamma$ and supported in $\Omega_{\epsilon}:=\{x \in \Omega$; $\operatorname{dist}(x, \Gamma)<\epsilon\}$, where $\epsilon>0$ is such that $\operatorname{Im} \Phi_{k} \geq \gamma x_{3}$ for $0 \leq x_{3} \leq \epsilon$ and $(x, \xi)$ in a small neighborhood of $\Sigma$ with some fixed $\gamma>0$ as above. Then we set

$$
u_{j}=\lambda_{j}^{1 / 2} \chi\left(H_{e}\left(\lambda_{j}\right) \psi_{j}+\tilde{u}_{j}\right) .
$$

Here $\tilde{u}_{j}=O\left(\lambda_{j}^{-\infty}\right)$ is a correction term chosen so that $N u_{j}=0$, where $N$ stands for the 1.h.s. of (1). It satisfies the condition $N \tilde{u}_{j}=-\lambda_{j}^{1 / 2} N_{e}\left(\lambda_{j}\right) \psi_{j}$, where the r.h.s. is $O\left(\lambda_{j}^{-\infty}\right)$ by our construction. It is not hard to show that $\tilde{u}_{j}$ can be chosen so that its $H^{2}$ norm is also $O\left(\lambda_{j}^{-\infty}\right)$ near $\Gamma$. With this choice of $u_{j}$ we have $u_{j} \in D(L)$.

We will show that

$$
C^{-1}\left\|\psi_{j}\right\|_{L^{2}(\Gamma)} \leq\left\|u_{j}\right\| \leq C\left\|\psi_{j}\right\|_{L^{2}(\Gamma)} \quad j=1,2, \ldots
$$

with some $C>1$. In fact, since $\psi_{j}$ are normalized, we have $\left\|\psi_{j}\right\|_{L^{2}(\Gamma)}=1$ above. In order to prove (19), integrate the estimate $\left\|\chi H_{e}\left(\lambda_{j}\right) \psi_{j}\right\|_{L^{2}\left(\Gamma_{t}\right)}^{2} \leq C e^{-2 \gamma t \lambda_{j}}\left\|\psi_{j}\right\|_{L^{2}(\Gamma)}^{2}$, where $\Gamma_{t}:=\left\{x_{3}=t\right\}$ with respect to $t \in(0, \epsilon)$ to get $\left\|\chi H_{e}\left(\lambda_{j}\right) \psi_{j}\right\|_{L^{2}\left(\Omega_{\epsilon}\right)} \leq C \lambda_{j}^{-1 / 2}\left\|\psi_{j}\right\|_{L^{2}(\Gamma)}$. This proves the second part of (19). In particular we get that $u_{j} \in D(L)$ and

$$
\left(L-\lambda_{j}^{2}\right) u_{j}=O\left(\lambda_{j}^{-\infty}\right), \quad u_{j} \in D(L),
$$


because the cut-off represented by $\chi$ occurs in $0<\epsilon_{1}<\operatorname{dist}(x, \Gamma)<\epsilon$, where $H_{e}\left(\lambda_{j}\right) \psi_{j}$ are exponentially small in $H^{1}$.

The first part of (19) follows from the trace theorem. Indeed, for a fixed $\epsilon>0$,

$$
\left\|\psi_{j}\right\|_{H^{3 / 2}(\Gamma)} \leq C \lambda_{j}^{-1 / 2}\left\|u_{j}\right\|_{H^{2}\left(\Omega_{\varepsilon}\right)}+O\left(\lambda_{j}^{-\infty}\right) \leq C \lambda_{j}^{3 / 2}\left\|u_{j}\right\|_{L^{2}\left(\Omega_{2 \varepsilon}\right)}+O\left(\lambda_{j}^{-\infty}\right),
$$

because $u_{j}$ solve $\left(L-\lambda_{j}^{2}\right) u_{j}=O\left(\lambda_{j}^{-\infty}\right)$ near $\Gamma$ and $L$ is elliptic. On the other hand, $\lambda_{j}^{3 / 2} \leq$ $C\left\|\phi_{j}\right\|_{H^{3 / 2}(\Gamma)}$ because $\phi_{j}$ are normalized eigenfunction of $\mathcal{P}$, and since $\psi_{j}=\phi_{j} /\left\|\phi_{j}\right\|, \mid \psi_{j} \|=$ $O(1)$, we get the same estimate for $\psi_{j}$. This, combined with (20) proves the first part of (19).

We are still not ready to apply the results in [S] to prove Theorem 1.1, because $u_{j}$, $j=1, \ldots$ are not necessarily asymptotically orthogonal to each other. However, they are linearly independent and we will show below that this property is preserved under $O\left(\lambda^{-\infty}\right)$ perturbations (in fact, $O\left(\lambda^{-4}\right)$ suffices). This would be enough to apply the results in [S] since it is easy to see from the proof of the main results in [S] (see [S, Lemma 4] and the arguments after it) that one can relax the orthogonality condition to the above property. We will not use this argument however, instead we will repeat some of the arguments in the proof of Theorem 2 in [S] adapted to the present situation.

Since we have standard Weyl asymptotic for the quasimodes $\lambda_{j}$ (eigenvalues of $\mathcal{P}$ ), we can group them into clusters. More precisely, fix $k$ large enough. Then we can find a sequence $J_{l}$ of intervals with the following properties: $\left|J_{l}\right|=O\left(\lambda^{3-k}\right), \lambda \in J_{l} ; \operatorname{dist}\left(J_{l}, J_{l+1}\right)=O\left(\lambda^{-k}\right)$, $\lambda \in J_{l}$; each $J_{l}$ contains $m_{l}=O\left(\lambda^{3}\right), \lambda \in J_{l}$ quasimodes staying at a distance at least $O\left(\lambda^{-k}\right)$, $\lambda \in J_{l}$ form the endpoints. We aim to prove that in

$$
\mathcal{U}_{l}:=\left\{\lambda \in \mathbf{C} ; \operatorname{Re} \lambda \in J_{l}, 0<\operatorname{Im} \lambda<S(\operatorname{Re} \lambda)\right\}
$$

there are at least $m_{l}$ resonances counted with multiplicities. Here $0<S(t)=O\left(t^{-\infty}\right), t \rightarrow \infty$ is a suitable chosen function.

Denote by $\Pi_{l}$ the projector onto the space spanned by the functions in the range of all residua of $R_{\chi}(\lambda)$ with poles in $\mathcal{U}_{l}$ with some fixed cut-off function $\chi$. Note that the residua of $R_{\chi}(\lambda)$ and those of the meromorphic continuation of $z \mapsto(L-z)^{-1}, z=\lambda^{2}$ have the same range. It follows from $\left[\mathrm{S}\right.$, Remark 6] that $\Pi_{l} u_{j}=u_{j}+O\left(\lambda_{j}^{-\infty}\right)$ for $j=1, \ldots, m_{l}$ if $S(t)$ is chosen in a suitable way. Functions $u_{j}, j=1, \ldots, m_{l}$ are linearly independent, because $V^{-1}\left(\lambda_{j}\right)\left(\left.u_{j}\right|_{\Gamma}\right)$ are asymptotically orthogonal. We aim to show that $\Pi_{l} u_{j}, l$-fixed, are also linearly independent. That would prove that $\operatorname{Rank}_{l} \geq m_{l}$ and the lower bound would follow from this as in [S].

To prove the linear independence of $\Pi_{l} u_{j}$, we will modify the $L^{2}$ structure near the boundary in order to make $u_{j}$ orthogonal. Choose $\|\cdot\|_{*}$ as follows (see (18))

$$
\left\|u_{j}\right\|_{*}=\lambda_{j}^{-1 / 2}\left\|\left.V^{-1}\left(\lambda_{j}\right) u_{j}\right|_{\Gamma}\right\|_{L^{2}(\Gamma)} \quad j=1,2, \ldots
$$

This norm induces a scalar product on $\mathcal{U}:=\operatorname{span}\left(u_{1}, u_{2}, \ldots\right)$. The norms $\|\cdot\|_{*}$ and the regular $L^{2}$ norm $\|\cdot\|$ are topologically equivalent on $\mathcal{U}$ because of the estimate (19). 
Let us extend the definition of the scalar product $(\cdot, \cdot)_{*}$ on the whole $L^{2}(\Omega)$ by setting $\left(u_{1}+v_{1}, u_{2}+v_{2}\right)_{*}:=\left(u_{1}, u_{2}\right)_{*}+\left(v_{1}, v_{2}\right)$, where $u_{1,2} \in \mathcal{U}, v_{1,2} \in \mathcal{U}^{\perp}$. Then $\|\cdot\|_{*}$ remains equivalent to $\|\cdot\|$.

Now, $\left(u_{i}, u_{j}\right)_{*}=\delta_{i j}+O\left(\lambda_{j}^{-\infty}\right), \forall i, j$ and $\Pi_{l} u_{j}=u_{j}+O\left(\lambda_{j}^{-\infty}\right), j=1, \ldots, m_{l}$. This allows us to complete the proof of Theorem 1.1 in the same way as in [S] in order to show that $\Pi_{l} u_{j}, j=1, \ldots, m_{l}$ are linearly independent, hence $\operatorname{Rank}_{l} \geq m_{l}$ for any $l$ large enough. Thus we conclude that Theorem 2 in [S] holds in this case as well and therefore

$$
N(r) \geq N_{\mathcal{P}}\left(r-r^{-k}\right)-C_{k}
$$

with $k \geq 1$. Here $N_{\mathcal{P}}(r)$ is the counting function of the eigenvalues of $\mathcal{P}$. The classical Weyl asymptotics imply that

$$
N_{\mathcal{P}}(r)=\frac{\operatorname{Area}(\Gamma)}{4 \pi c_{R}^{2}} r^{2}+O(r)
$$

Those two inequalities imply immediately the estimate in Theorem 1.1.

Tracing back the construction of the operator $V$ in Propositions 2.2, 3.1, 3.2, we get that

$$
\varphi_{j}=\mathcal{A} \phi_{j}
$$

where $\mathcal{A}$ is a vector-valued $\lambda$ - $\Psi \mathrm{DO}$ with principal symbol $\sigma_{p}(A)=\left(a_{1}^{\prime}\right)^{-1 / 2} v_{1}$ (see Proposition 2.1). Since $a_{1}^{\prime}$ depends on $|\xi|_{x}$ only and $\mathrm{WF}_{\lambda}\left(\left\{\phi_{j}\right\}\right) \subset \Sigma$, we can replace $a_{1}^{\prime}$ above by the constant $\left.a_{1}^{\prime}\right|_{\Sigma}$ and that will result in an error term $O\left(\lambda_{j}^{-1}\right)$ in $(21)$. By Proposition 2.1 we therefore get

$$
\varphi_{j}=\left(1-\frac{c_{R}^{-2}}{2 \mu_{0}}\right) \lambda_{j}^{-1} \nabla_{\Gamma} \phi_{j}+\left.\beta\right|_{\Sigma} n(x) \phi_{j}+O\left(\lambda_{j}^{-1}\right)
$$

modulo a normalizing factor independent of $j$. Clearly, we have the same for $\psi_{j}$. This proves the last part of Theorem 1.2. Note that $u_{j}$ in Theorem 1.2 should satisfy $\left\|u_{j}\right\|=1$, while we have $\left\|u_{j}\right\|_{*}=1+O\left(\lambda_{j}^{-\infty}\right)$, but we can always normalize $u_{j}$ by keeping their properties in view of the equivalence of $\|\cdot\|$ and $\|\cdot\|_{*}$.

Proof of Theorem 1.3 As mentioned above, if $\lambda_{0}$ is not a Dirichlet resonance, then $\lambda_{0}$ is a (Neumann) resonance if and only if it is a pole of the inverse $\mathcal{N}^{-1}(\lambda)$ [SV1] and its multiplicity is given by $[\mathrm{SjV}]$

$$
\operatorname{mult}\left(\lambda_{0}\right)=\operatorname{tr} \frac{1}{2 \pi i} \oint_{\gamma} \mathcal{N}^{-1}(\lambda) \dot{\mathcal{N}}(\lambda) d \lambda
$$

Here $\dot{\mathcal{N}}$ denotes derivative w.r.t. $\lambda$ and $\gamma$ is a small positively oriented closed curve such that there are no other poles of $N^{-1}$ in its interior. Since we assume here that $\mathcal{O}$ is strictly convex, there are no Dirichlet resonances in $\Lambda$.

Part (a) of Theorem 1.3 is essentially proved in $[\mathrm{SjV}]$. We recall the proof below because we need it in order to complete the proof of part (b). Recall that $\lambda$ in our calculus belongs to the strip $0 \leq \operatorname{Im} \lambda \leq 1$, $\operatorname{Re} \lambda>0$. As shown in $[\mathrm{SjV}]$, if we can find $N \in L^{1,1}$ (denoted there 
by $h P(h), h=1 / \lambda)$, self-adjoint for real $\lambda$, such that $(\mathcal{N}-N) X \in L^{-\infty,-\infty}, X(\mathcal{N}-N) \in$ $L^{-\infty,-\infty}$ for $X$ with $\mathrm{WF}_{\lambda}(X)$ in a neighborhood of $\Sigma$, then there is a bijection between resonances near the real axis and the poles of $N^{-1}(\lambda)$ with the desired properties. More precisely, one can divide the poles $\lambda$ of $N^{-1}$ with $\lambda$ sufficiently large into groups (clusters), each one contained in an interval $J_{l}$ depending also on a parameter $k \gg 1$, such that those intervals do not overlap and $\left|J_{l}\right|=O\left(\lambda^{3-k}\right), \lambda \in J_{l}$. Next, $\left\|N^{-1}\right\|=O\left(\lambda^{1-k}\right)$ for $\lambda \notin J_{l}$. The number of such intervals in $(\lambda / 2, \lambda)$ is $O\left(\lambda^{2}\right)$. Next, the number of resonances in

$$
\mathcal{U}_{l}:=\left\{\operatorname{Re} \lambda \in J_{l} ;|\operatorname{Im} \lambda| \leq(\operatorname{Re} \lambda)^{1-k}\right\}
$$

is equal to the number of poles of $N^{-1}$ there, counted with multiplicities. Arguing as above, we can choose $N=V(\lambda) \operatorname{diag}(\mathcal{P}-\lambda, Q(\lambda)) V^{*}(\lambda)$. Then for $|\lambda|$ large enough the poles of $N(\lambda)$ are actually the eigenvalues of $\mathcal{P}$ counted with multiplicities. So the intervals $J_{l}$ can be constructed as in the proof of Theorem 1.1 above but instead of a lower bound, we have an equality of the number of resonances and quasimodes in $\mathcal{U}_{l}, l \gg 1$. Varying $k$, we thus prove as in $[\mathrm{SjV}]$ that there exists a bijection from the set of the eigenvalues of $\mathcal{P}$ to all resonances in $\Lambda_{S}:=\Lambda \cap\{0<\operatorname{Im} \lambda<S(\operatorname{Re} \lambda)\}$ with some $S(t)=O\left(t^{-\infty}\right)$ as $t \rightarrow \infty$ and this bijection satisfies the estimate in Theorem 1.3(a). By [SV1], all resonances $\rho_{j} \in \Lambda$ with sufficiently large real parts satisfy $0<\operatorname{Im} \rho_{j}=O\left(\left(\operatorname{Re} \rho_{j}\right)^{-\infty}\right)$, so we can pick $S(t)$ above in a suitable way to make sure that all sufficiently large resonances in $\Lambda$ are actually in $\Lambda_{S}$.

It remains to prove estimate (2) in Theorem $1.3(\mathrm{~b})$. Let $\Pi_{l} u_{j}, j=1, \ldots, m_{l}$ be as above with $l$ fixed. Then $\Pi_{l} u_{j}=u_{j}+O\left(\lambda_{j}^{-\infty}\right)$. By characterizing resonances as the eigenvalues of a certain complex scaled operator $L_{\theta}$, it follows from [SjZ] that the rank of $\Pi_{l}$ is a sum of the ranks of the residue of $R_{\chi}(\lambda)$ at each one of the distinct resonances among $\rho_{j}, j=1, \ldots, m_{l}$. It is enough to prove (2) for $v_{j}:=\Pi_{l} u_{j}, j=1, \ldots, m_{l}, l \gg 1$ since for any $l$ the functions $v_{j}$ form a basis in $\operatorname{Rank} \Pi_{l}$, orthonormal up to an error $O\left(\left|\rho_{j}\right|^{-\infty}\right), j=1, \ldots, m_{l}$ with respect to the scalar product $(\cdot, \cdot)_{*}$. Estimate (2) holds for $u_{j}$, thus it is enough to prove that $v_{j}=u_{j}+O\left(\lambda_{j}^{-1}\right)$ in $H^{1 / 2+\epsilon}(\Omega), \epsilon>0$. Recall that both $v_{j}$ and $u_{j}$ are supported in a fixed compact neighborhood of $\Gamma$. Then (2) would follow from the trace theorem. Tracing the arguments in the proof of Theorem 1 in [S] (see also [TZ]), we see that we have actually $v_{j}=u_{j}+O\left(\lambda_{j}^{-\infty}\right)$ in $H^{2}(\Omega)$. This follows from the fact that inequalities (11) and (13) in [S] remain true if the operator norm is considered in $\mathcal{B}\left(L^{2}, H^{2}\right)$ with right-hand sides multiplied by $C h^{-2}$.

This completes the proof of Theorem 1.3.

\section{References}

[CP] F. Cardoso and G. Popov, Rayleigh quasimodes in linear elasticity, Comm. P.D.E. 17(1992), 1327-1367.

[CV1] F. Cardoso And G. Vodev, Asymptotic behaviour of the scattering phase in linear elasticity for a strictly convex body, Comm. P.D.E. 22(1997), no. 11-12, 2025-2049. 
[CV2] F. Cardoso And G. Vodev, Asymptotic behaviour of the scattering phase in linear elasticity II, Osaka J. Math. 35(1998), no. 2, 397-405.

[G] C. GÉRARD, Asymptotique des poles de la matrice de scattering pour deux obstacles strictement convex, Bull. Soc. Math. France, Mémoire n. 31, 116, 1988.

[K1] M. KAWASHITA, On the local-energy decay property for the elastic wave equation with the Neumann boundary conditions, Duke Math. J. 67(1992), 333-351.

[K2] M. Kawashita, On a region free from the poles of the resolvent and decay rate of the local energy for the elastic wave equation, Indiana Univ. Math. J. 43(1994), no. 3, 1013-1043.

[N] G. NAKAmura, Existence and propagation of Rayleigh waves and pulses, in: Modern theory of anisotropic elasticity and applications (Research Triangle Park, NC, 1990), 215-231, SIAM, Philadelphia, PA, 1991.

[P] G. Popov, Quasimodes for the Laplace operator and glancing hypersurfaces, In: M.Beals, R.Melrose, J.Rauch (eds): Proceeding of Conference on Microlocal Analysis and nonlinear waves, Minessota 1989, Springer, 1991.

[PV] G. Popov And G. Vodev, Resonances near the real axis for transparent obstacles, Comm. Math. Phys., to appear.

[R] LoRd RAYleigh, On waves propagated along plane surface of an elastic solid, Proc. London Math. Soc. 17(1885), 4-11.

[SjV] J. SuÖstrand And G. Vodev, Asymptotics of the number of Rayleigh resonances, Math. Ann. 309(1997), 287-306.

[SjZ] J. SuÖstrand And M. ZwOrski, Complex scaling and the distribution of scattering poles, Journal of AMS 4(4)(1991), 729-769.

[SV1] P. Stefanov And G. Vodev, Distribution of resonances for the Neumann problem in linear elasticity outside a strictly convex body, Duke Math. J. 78(1995), 577-714.

[SV2] P. Stefanov And G. Vodev, Neumann resonances in linear elasticity for an arbitrary body, Comm. Math. Phys. 176(1996), 645-659.

[S] P. Stefanov, Quasimodes and resonances: sharp lower bounds, Duke Math. J. 99(1999), 75-92.

[TZ] S.-H. TAng And M.Zworski, From quasimodes to resonances, Math. Res. Lett., 5(1988), 261-272.

[T1] M. TAYLOR, Rayleigh waves in linear elasticity as a propagation of singularities phenomenon, in Proc. Conf. on P.D.E. and Geometry, Marcel Dekker, New York, 1979, 273-291. 
[T2] M. TAYlor, Pseudodifferential Operators, Princeton University Press, Princeton, 1981.

[V] G. Vodev, Existence of Rayleigh resonances exponentially close to the real axis, Ann. Inst. H. Poincaré (Phys. Théor.) 67(1997), no. 1, 41-57.

[Y] K. Yамамото, Singularities of solutions to the boundary value problems for elastic and Maxwell's equations, Japan J. Math. 14(1988), 119-163. 